\title{
Update on Biologics for Psoriasis in Clinical Practice
}

\author{
Mirjana G. Ivanic, BA; Grace S. Ahn, BS; Patrick Herndon, BS; Jashin J. Wu, MD
}

\section{PRACTICE POINTS}

- Choosing a biologic best fit for each patient's individual health profile can reduce psoriasis disease burden.

- Clinicians should educate psoriasis patients that biologics are safe for most comorbidities, and conditions such as obesity have been associated with poorer therapeutic response.

- It is important to discuss possible side effects of biologics with patients and reassure them that mild side effects are the most common during therapy.

Biologics have impacted the clinical management of moderate to severe psoriasis. This review article highlights new data findings from phase 3 clinical trials $(N=8)$ published between May 2020 and February 2021. Data on the efficacy of US Food and Drug Administration-approved biologics for treating psoriasis affirms durable skin clearance in the presence of comorbidities and after treatment gaps. This article aims to provide clinicians with up-to-date knowledge on biologic performance focusing on skin disease clearance, time to skin disease clearance, loss of response and relapse, and treatment-emergent adverse events (TEAEs). Recent trial data in this review focus on treatment with IL-17A inhibitors and IL-23 inhibitors.

Cutis. 2021;108(suppl 2):15-18.

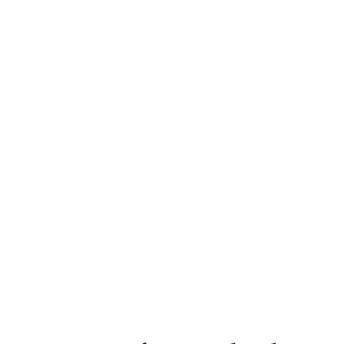

B iologics have transformed the management of moderate to severe psoriasis. There currently are 11 biologics approved by the US Food and Drug Administration (Table) for psoriasis treatment that have been affirmed by various clinical studies. This article provides dosing initiation, maintenance information, and updated clinical data using phase 3 studies $(\mathrm{N}=8)$ published between May 2020 and February 2021. Generic names of the 11 biologics were searched separately in the PubMed database within the specified date range. Subsequent results were reviewed by title and selected for phase 3 and 4 trials. Clinical data in this review focus on reducing patient disease burden by allocating a biologic best fit for each patient's individual health profile.

\section{IL-17A Inhibitors Update}

Secukinumab is safe and efficacious for skin clearance in the presence of comorbidities and can be used for improving plaque psoriasis and palmoplantar pustular psoriasis. An extension of a phase 3 randomized controlled trial (RCT)-2PRECISE-evaluated the efficacy and safety of secukinumab dosing at $300 \mathrm{mg}(\mathrm{n}=79)$ and $150 \mathrm{mg}(\mathrm{n}=80)$ in adults with moderate to severe palmoplantar pustular psoriasis (palmoplantar psoriasis area and severity index [PPPASI] score $\geq 12$ and dermatology life quality index [DLQI] $\geq 10$ ) over 148 weeks. ${ }^{1}$ Extension

Ms. Ivanic is from Meharry Medical College, School of Medicine, Nashville, Tennessee. Ms. Ahn is from the Department of Dermatology, University of California San Diego School of Medicine. Mr. Herndon is from Oakland University William Beaumont School of Medicine, Rochester, Michigan.

Dr. Wu is from Dermatology Research and Education Foundation, Irvine, California.

Ms. Ivanic, Ms. Ahn, and Mr. Herndon report no conflict of interest. Dr. Wu is or has been an investigator, consultant, or speaker for AbbVie, Almirall, Amgen, Arcutis, Aristea Therapeutics, Bausch Health (Ortho Dermatologics), Boehringer Ingelheim, Bristol-Myers Squibb, Dermavant, Dr. Reddy's Laboratories, Eli Lilly, Galderma, Janssen, LEO Pharma, Mindera, Novartis, Regeneron, Sanofi Genzyme, Solius, Sun Pharmaceutical, UCB, and Zerigo Health.

Correspondence: Jashin J. Wu, MD (jashinwu@gmail.com).

doi: 10.12788 /cutis.0317 


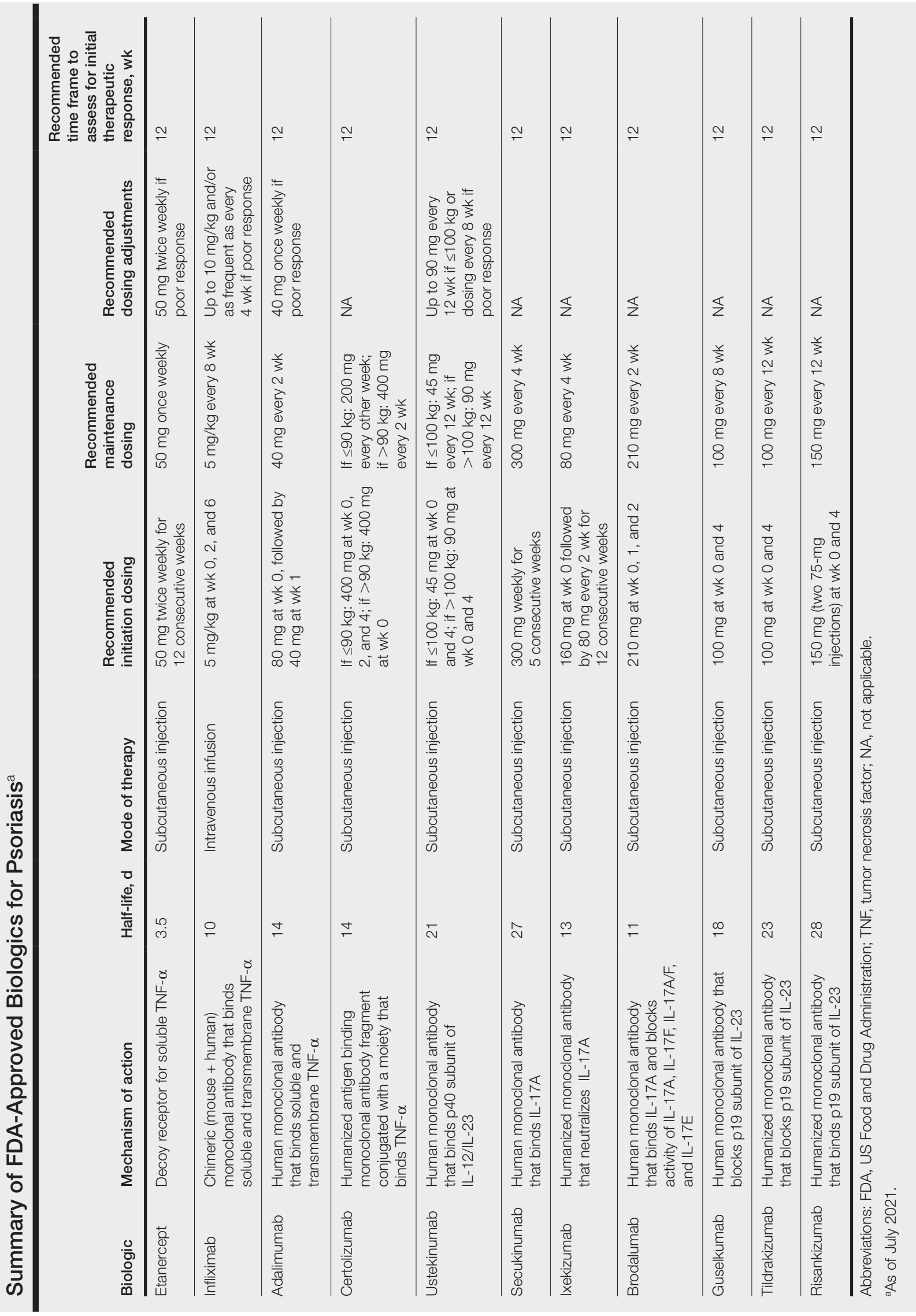


patients were included from the 52-week 2PRECISE study per the investigator's judgement of a meaningful clinical response (exact criteria not described). All treatment groups demonstrated a mean (SD) PPPASI of 22.7 (9.5) by the extension trial's start. Results affirmed that clinical response waned after week 148 in all groups excluding placebo/secukinumab $150 \mathrm{mg}$, which maintained a mean (SD) PPPASI of 22.7 (9.5). The most frequent adverse events were nasopharyngitis, pustular psoriasis, headache, and pruritus. ${ }^{1}$

Comorbidities do not have a major impact on secukinumab's efficacy. A post hoc analysis of 4 phase 3 RCTs-ERASURE, FIXTURE, FEATURE, and JUNCTURE-gathered data from adult patients $(\mathrm{N}=2401)$ to assess baseline comorbidities with efficacy and safety of secukinumab vs etanercept after 12 weeks of treatment. ${ }^{2}$ Sixty-one percent $(n=1469)$ had at least 1 comorbidity, most frequently obesity, hypertension, psoriatic arthritis, hyperlipidemia, or diabetes mellitus. All patient groups had a greater likelihood of a psoriasis area and severity index (PASI) response with any dose of secukinumab vs patients with comorbidities who were taking etanercept or placebo $(P<.05)$ at week 12 . All groups had a greater likelihood of achieving investigator global assessment scores of 0/1 (clear/almost clear) vs patients with comorbidities taking etanercept or placebo $(P<.05)$. Baseline comorbidities did not significantly affect treatment response, except obesity, which was associated with decreased probability of achieving all PASI and investigator global assessment $(P<.01)$ responses. Secukinumab-treated patients with and without comorbidities had equivalent likelihood of treatment-emergent adverse events (TEAEs). ${ }^{2}$

Brodalumab is an effective biologic that has shown long-term safety with continuous administration. Continuous brodalumab and brodalumab after placebo demonstrated impactful skin clearance after 120 weeks in AMAGINE-1, a phase 3 RCT involving adults $(\mathrm{N}=442)$ with moderate to severe plaque psoriasis. ${ }^{3}$ Patients randomized to brodalumab $210 \mathrm{mg}(\mathrm{n}=222)$ or placebo $(n=220)$ were rerandomized according to initial treatment response. In patients switching from brodalumab to placebo at week $12,55 \%$ and $94 \%$ achieved PASI 75 at week 20 and week 120, respectively, and 75\% reached PASI 100 at week 120. Of patients with static physician global assessment (sPGA) scores of 0/1 (clear/almost clear) at week 12 who were rerandomized to brodalumab, $96 \%$ and $80 \%$ (using observed data) achieved PASI 75 and PASI 100, respectively. Mean (SD) time to return of skin disease following withdrawal of brodalumab was 74.7 (50.5) days. Treatment-emergent adverse events included headaches, arthralgia, diarrhea, and nausea. Suicidal ideation was rare (this study had 1 completed suicide), and authors cited that no causal association has been made between brodalumab and suicidality. Brodalumab also demonstrated favorable treatment response in patients who underwent a lapse in treatment, offering real-world value, as intermittent treatment administration can occur because of personal or financial reasons. ${ }^{3}$

Ixekizumab is associated with more rapid skin clearance, better resolution of nail psoriasis, and superior improvement in quality-of-life measures when compared with guselkumab. The phase 3 study IXORA-R compared skin and nail clearance as well as patient-reported outcomes over 24 weeks with ixekizumab $80 \mathrm{mg}(\mathrm{n}=520)$ vs guselkumab $100 \mathrm{mg}(\mathrm{n}=507)$ in adults with moderate to severe plaque psoriasis. ${ }^{4}$ Ixekizumab (50\%) was shown to be no worse than guselkumab (52\%; difference, $-2.3 \%$ ) using a noninferiority test (noninferiority margin of $-11.4 \%$ ). The treatments exhibited similar efficacy, with no significant difference in proportion of patients reaching PASI $100(P=.41)$. Ixekizumab patients tended to have skin clearance sooner than guselkumab patients, reaching PASI 50/75/90 and PASI 100 in a median time that was 2 weeks and 7.5 weeks earlier, respectively. More ixekizumab patients (52\%) achieved clear nails vs guselkumab patients (31\%; $P=.007)$. Ixekizumab patients reported greater satisfaction with their skin disease affecting quality of life (DLQI), with more DLQI 0/1 (no effect at all on patient's life) scores and being itch free $(P<.05)$. Ixekizumab was associated with significantly more days of complete skin clearance (PASI 100) vs guselkumab (55.6 days vs 42.2 days; $P<.001)$. Although an upper respiratory tract infection was the most common TEAE, the proportion of TEAEs was similar between treatments. ${ }^{4}$

\section{IL-23 Inhibitors Update}

Tildrakizumab has similar long-term skin clearance efficacy and safety in patients with psoriasis with and without comorbid metabolic syndrome (MetS). A post hoc analysis of 2 phase 2 RCTs (reSURFACE 1/2) involving adults $(\mathrm{N}=338$ and $\mathrm{N}=307)$ with moderate to severe plaque psoriasis assessed long-term efficacy (3 years), drug survival, and safety for 5 years of continuous tildrakizumab $100 \mathrm{mg}$ and $200 \mathrm{mg}$ in adults with comorbid MetS. ${ }^{5}$ Although no difference in efficacy was concluded, greater body mass index of the MetS population was shown to be associated with lower biologic efficacy compared to the general population. The proportion of patients who achieved PASI 75 at week 52 was comparable in patients with MetS and patients without MetS (tildrakizumab $100 \mathrm{mg}$, 85\% and $86 \%$ vs $86 \%$ and $94 \%$ for reSURFACE $1 / 2$, respectively; tildrakizumab $200 \mathrm{mg}$, $76 \%$ and $87 \%$ vs $76 \%$ and $87 \%$ for reSURFACE $1 / 2$, respectively). ${ }^{5}$

Tildrakizumab also demonstrated efficacy and safety for up to 5 years in 2 other phase 3 RCTs with no dose-related differences in frequency of injections and malignancies. Tildrakizumab $100 \mathrm{mg}$ is the recommended dose. The 200-mg dose can be utilized in patients with a high burden of disease and disability. reSURFACE 1 and reSURFACE 2 involved adults with chronic moderate to severe plaque psoriasis randomized to tildrakizumab $100 \mathrm{mg}$, $200 \mathrm{mg}$, or placebo with the option of long-term extension to week 244 if patients reached $50 \%$ or greater improvement 
from baseline PASI score. ${ }^{6}$ Patients in reSURFACE 2 also were randomized to etanercept $50 \mathrm{mg}$ with partial responders and nonresponders at week 28 switching to tildrakizumab $200 \mathrm{mg}$ until week 244. Extension results showed PASI 75 achievement in $88.7 \%$ (95\% CI, 84.6\%-92.1\%) of patients taking tildrakizumab $100 \mathrm{mg}(\mathrm{n}=235), 92.5 \%$ (95\% CI, 88.1\%-95.7\%) of patients taking tildrakizumab $200 \mathrm{mg}(\mathrm{n}=176)$, and $81.3 \%$ (95\% CI, 72.6\%-88.2\%) of patients taking etanercept/partial nonresponders $(n=85)$. The most common TEAE was nasopharyngitis (10.5/100 patient-years for tildrakizumab $100 \mathrm{mg}$ and 10.7/100 patient-years for tildrakizumab $200 \mathrm{mg}$ ). The frequency of severe infections (eg, diverticulitis, pneumonia, cellulitis, appendicitis) was 1.2 per 100 patient-years for tildrakizumab $100 \mathrm{mg}$ and 1.3 per 100 patient-years for tildrakizumab $200 \mathrm{mg} .{ }^{6}$

Risankizumab and tildrakizumab require the lowest number of injections, thereby providing sustainable skin clearance with a convenient injection dosing schedule for patients. Risankizumab efficacy $(8.2 \%$ with inferiority margin of $12 \%$ ) was noninferior to secukinumab when assessing the proportion of PASI 90 responders at week 16 (after 2 doses of risankizumab vs 7 doses of secukinumab). ${ }^{7}$ IMMerge, an international phase 3 RCT, involved adults $(\mathrm{N}=327)$ with moderate to severe plaque psoriasis to compare the safety and efficacy of risankizumab $150 \mathrm{mg}(\mathrm{n}=164)$ vs secukinumab $300 \mathrm{mg}(\mathrm{n}=163)$ up to 52 weeks. A greater proportion of the risankizumab arm (86.6\%) achieved PASI 90 in 52 weeks compared to the secukinumab arm (57.1\%). Superior skin clearance (PASI 90) at week 52 was achieved after 5 doses with risankizumab vs 16 doses of secukinumab. Risankizumab TEAEs were nasopharyngitis, upper respiratory tract infection, headache, arthralgia, diarrhea, and bronchitis. ${ }^{7}$

Continuous risankizumab treatment shows substantially stronger skin clearing performance compared with intermittent treatment following drug withdrawal, demonstrating that treatment gaps minimize therapeutic response. IMMhance, an international phase $3 \mathrm{RCT}$ involving adults $(\mathrm{N}=507)$ with moderate to severe plaque psoriasis, evaluated the safety and efficacy with risankizumab $150 \mathrm{mg}$ after 52 weeks and 104 weeks. ${ }^{8}$ Part A randomized patients to risankizumab $150 \mathrm{mg}(\mathrm{n}=407)$ or placebo $(n=100)$. Part B rerandomized patients at week 28 to continue risankizumab $150 \mathrm{mg}$ or placebo (designated as withdrawal of treatment; later re-treated with risankizumab $150 \mathrm{mg}$ if patients had sPGA $\geq 3$ ). At week 52 , significantly more patients reached sPGA score of $0 / 1$ with risankizumab/risankizumab $(n=97[87.4 \%])$ vs risankizumab/placebo $(\mathrm{n}=138$ [61.3\%]; $P<.001)$. At week 104, significantly more patients reached an sPGA score of 0/1 with risankizumab/risankizumab
( $\mathrm{n}=90[81.1 \%])$ vs risankizumab/placebo $(\mathrm{n}=16$ [7.1\%]; $P<.001$ ). Risankizumab exhibited longevity following withdrawal, as median time to loss of response and relapse was 42 weeks (sPGA $\geq 3$ ). The extent of TEAEs was similar between risankizumab and placebo and included nasopharyngitis, upper respiratory tract infection, headache, and back pain. ${ }^{8}$

\section{Final Thoughts}

Biologics for psoriasis help produce intended results for skin disease clearance and are tools for precision medicine. Recent data demonstrate safe, durable, and continuous efficacy with biologics, which offer patients a better chance of treatment success. This guide may serve as a quick reference for biologic selection with special consideration of individual disease characteristics and comorbidities.

\section{REFERENCES}

1. Mrowietz U, Bachelez $\mathrm{H}$, Burden AD, et al. Efficacy and safety of secukinumab in moderate to severe palmoplantar pustular psoriasis over 148 weeks: extension of the 2PRECISE study. J Am Acad Dermatol. 2021;84:552-554. doi:10.1016/j.jaad.2020.06.038

2. Gottlieb AB, Wu JJ, Griffiths CEM, et al. Clinical efficacy and safety of secukinumab in patients with psoriasis and comorbidities: pooled analysis of 4 phase 3 clinical trials [published online October 21, 2020]. J Dermatolog Treat. doi:10.1080/09546634.2020.1832187

3. Papp K, Menter A, Leonardi C, et al. Long-term efficacy and safety of brodalumab in psoriasis through 120 weeks and after withdrawal and retreatment: subgroup analysis of a randomized phase III trial (AMAGINE-1). Br J Dermatol. 2020;183:1037-1048. doi:10.1111/ bjd.19132

4. Blauvelt A, Leonardi C, Elewski B, et al. A head-to-head comparison of ixekizumab vs. guselkumab in patients with moderate-to-severe plaque psoriasis: 24-week efficacy and safety results from a randomized, double-blinded trial. Br J Dermatol. 2021;184:1047-1058. doi:10.1111/ bjd.19509

5. Lebwohl MG, Leonardi CL, Mehta NN, et al. Tildrakizumab efficacy, drug survival, and safety are comparable in patients with psoriasis with and without metabolic syndrome: long-term results from 2 phase 3 randomized controlled studies (reSURFACE 1 and reSURFACE 2). J Am Acad Dermatol. 2021;84:398-407. doi:10.1016/j .jaad.2020.09.047

6. Thaci D, Piaserico S, Warren RB, et al. Five-year efficacy and safety of tildrakizumab in patients with moderate-to-severe psoriasis who respond at week 28: pooled analyses of two randomized phase III clinical trials (reSURFACE 1 and reSURFACE 2)[published online February 5, 2021]. Br J Dermatol. doi:10.1111/bjd.19866

7. Warren RB, Blauvelt A, Poulin Y, et al. Efficacy and safety of risankizumab vs. secukinumab in patients with moderate-to-severe plaque psoriasis (IMMerge): results from a phase III, randomized, open-label, efficacy-assessor-blinded clinical trial. Br J Dermatol. 2021;184:50-59. doi:10.1111/bjd.19341

8. Blauvelt A, Leonardi CL, Gooderham M, et al. Efficacy and safety of continuous risankizumab therapy vs treatment withdrawal in patients with moderate to severe plaque psoriasis: a phase 3 randomized clinical trial. JAMA Dermatol. 2020;156:649-658. doi:10.1001/ jamadermatol.2020.0723 